(P2-97) The Differences Between Triage and Start that are Applied to Train Derailment A. Hashimoto, T. Ueda, J. Hirata, K. Kuboyama, T. Yamada, J. Kotani

Emergency and Critical Care, Nishinomiyashi, Japan

The derailment accident occurred on 25 April 2005. 562 passengers were injured and 107 people died. Based on our periodic training in mass disaster triage, we accepted 113 wounded victims regardless of the severity of their condition. Initially, Simple Triage and Rapid Treatment was supposed to be performed by an emergency physician as a triage officer but START is not the most effective method for this case because patients of different severity levels were admitted to our hospital. Therefore, we performed triage by inspection and palpation based on our experience and intuition. Patient's facial color, breathing patterns, and trauma were processed for inspection. Furthermore, palpation was carried on without counting pulse and we diagnosed victims based upon strength of pulse and sensation of cold. From inspirational and sensuous diagnosis, the severity was determined. Comparing START with experiential and intuitive triage, differences between level of consciousness, the severity of trauma, and vital sign were examined. In addition, which components were necessary for primary survey was also reviewed.

Method: Severe or moderate, which were evaluated from ISS, vital sign, and Revised Trauma Score: RTS, Probability of survival: PS, were classified and then agreement rate, sensitivity, and specificity between START (S model) and triage (D model) were assessed. For START, data was extracted from clinical records.

Result: Agreement rate between the S model and the D model was $76.9 \%$. Moreover, at the percentage of questions answered, the $\mathrm{S}$ model showed $84.2 \%$ and the D model showed $68.4 \%$. Thus the experiment showed significant difference. 5 over triage cases appeared when the D model was operated. RTS, vital sign, and PS did not show great differences.

Conclusion: It could be difficult and insufficient to use START when a mass disaster occurs, however experiential and intuitive triage is also effective as START.

Prehosp Disaster Med 2011;26(Suppl. 1):s168

doi:10.1017/S1049023X11005425

(P2-98) Disease Pattern in a Rural Setting Three Weeks After the 2008 Sichuan, China Earthquake-Hong Kong Red Cross Basic Health Clinic in Yanmen K. Hung, ${ }^{1}$ E.Y.Y. Chan, ${ }^{2}$ E. Lam, ${ }^{3}$ T. Rainer, ${ }^{1}$ C. Grabam ${ }^{1}$

1. Accident \& Emergency Medicine Academic Unit, NT, Hong Kong

2. School of Public Health, NT, Hong Kong

3. HK, Hong Kong

Background: The Sichuan earthquake on 12 May 2008 with a magnitude of 8.0 killed $>87,000$ people. The response of the Hong Kong Red Cross (HKRC) included a basic healthcare team to a small rural town called Yanmen three weeks after the earthquake. The objective of this study was to review HKRC's medical records to identify the disease pattern in the Chinese rural setting post earthquake.
Methods: A cross-sectional, record-based study of all patients treated by the HKRC basic healthcare team from 01 June to 19 June 2008. This retrospective study studied the medical records collected during the clinical consultations, and descriptive analysis was performed for the demographic and clinical information. Blood pressure measurement was classified according to the JNC-7 classification, and the age- and gender-specific prevalence of hypertension was sought.

Results: A total of 2,034 cases were seen during the 19-day period with daily attendance ranging from 73 to 153 cases. Musculoskeletal, respiratory, and gastrointestinal problems were the top three categories and accounted for 30.4, 17.4, and $12.7 \%$ respectively. Trauma accounted for $5.4 \%$ of the cases attended, and $26.0 \%$ of the injuries were related to the earthquake. A total of $38.7 \%$ of the consultations were for medical problems that existed before the earthquake. A total of $43.4 \%$ patients with blood pressure measurements were above the recognized cutoff for hypertension.

Conclusions: Due to the impact and time phase post disaster, trauma, and injuries directly resulting from the earthquake did not contribute to a large number of cases. However, the management of chronic illness was an important issue, especially with the excessive prevalence of high blood pressure found in this study. Further studies to investigate the relationship between chronic diseases including hypertension and postearthquake conditions are needed.

Prehosp Disaster Med 2011;26(Suppl. 1):s168

doi:10.1017/S1049023X11005437

\section{(P2-99) Russian-Chinese Collaboration for Disaster Risk Reduction}

G.V. Kipor, S.F. Goncharov, N.K. Pichugina

Administration, Moscow, Russian Federation

The Disaster Medicine Centre "Zaschita" is a coordinating center with the general goal of management and medical relief delivery in response to major emergencies. The Russian Disaster Medicine Service has accumulated a national and international experiences of emergency responses to earthquakes (Columbia 1999, Neftegorsk 1995, Turkey 1999, Iran 2003, Afghanistan 2002, China 2008, etc.). The Health Ministries of the Russian Federation and of Peoples Republic of China have concluded a treaty of collaborative activities in the field of in public health services and disaster medicine. The promotion of cooperation in health sector is based on the key elements of collaboration, which is an a method coping with emergencies. All activities have been discussed during annual meetings, and are planned for participants on the basis of practical field experience and of emergency medical care technologies. These include: (1) mechanisms of trans-boundary field collaboration in management during major disasters; (2) sharing of field experiences for coping with natural disasters; (3) early warning networks and information technologies; (4) staff preparedness programs and postgraduate education; (5) new psycho-physiological technologies for staff selection, training, and conformity of medical teams; (6) coordination and management of international-scale exercises; (7) technology of medical relief provided for injured in major disasters; 